\title{
A Huge Ovarian Mucinous Cystadenoma, size matters
}

\author{
Abdulaziz Alobaid, \\ Women's specialized hospital, King Fahad, Medical City \\ Riyadh, Saudi Arabia
}

We Present a 53-year-old postmenopausal woman who presented with a huge abdominal-pelvic distention. Abdominal examination was remarkable for a cystic, diffuse, tense mass with extensive skin pressure ulcers. The abdominal circumference was $181 \mathrm{~cm}$.

Laboratory tests were remarkable for elevated CA 125 levels (298 U/mL). Abdominal- pelvic ultrasound revealed a huge multiloculated cyst occupying the whole abdomen with multiple thick septations and fine internal echoes. There was minimal free intraperitoneal fluid.

The patient had exploratory laparotomy. She remained on her right side throughout the entire procedure as it was difficult to mobilize the mass. Away from the pressure ulcers foci, a midline incision of about $35 \mathrm{~cm}$ in the healthy skin was performed. The mass was resected $(73 \times 51 \times 42 \mathrm{~cm})$ and 84 liters of mixed serous-mucinous fluid were drained. A frozen section biopsy was consistent with benign mucinous cystadenoma. The origin of the mass was identified to be the right ovary, and right salpingooophorectomy was done. The left ovary had a multilocular mass of $15 \mathrm{~cm}$ in diameter, and left salpingo-oophorectomy was performed. The surgery was uncomplicated. Histopathological examination confirmed the diagnosis of bilateral benign mucinous cystadenomas.

The patient was discharged home in good condition 60 days post-operatively. The pre- operative patient body weight was 172 $\mathrm{kg}$, and the postoperative weight was $64 \mathrm{~kg}$. At a postoperative follow-up in the outpatient clinic, the patient showed up in good condition.

To the best of our knowledge, we report one of the largest ovarian tumor (if not the largest ever) in the English medical literature. Also, we report that it is technically feasible to manage an extremely large-sized mass with satisfactorily perioperative outcomes. This should be done through a multidisciplinary approach that demands an orchestrated collaboration between different specialists to yield an optimized perioperative care.
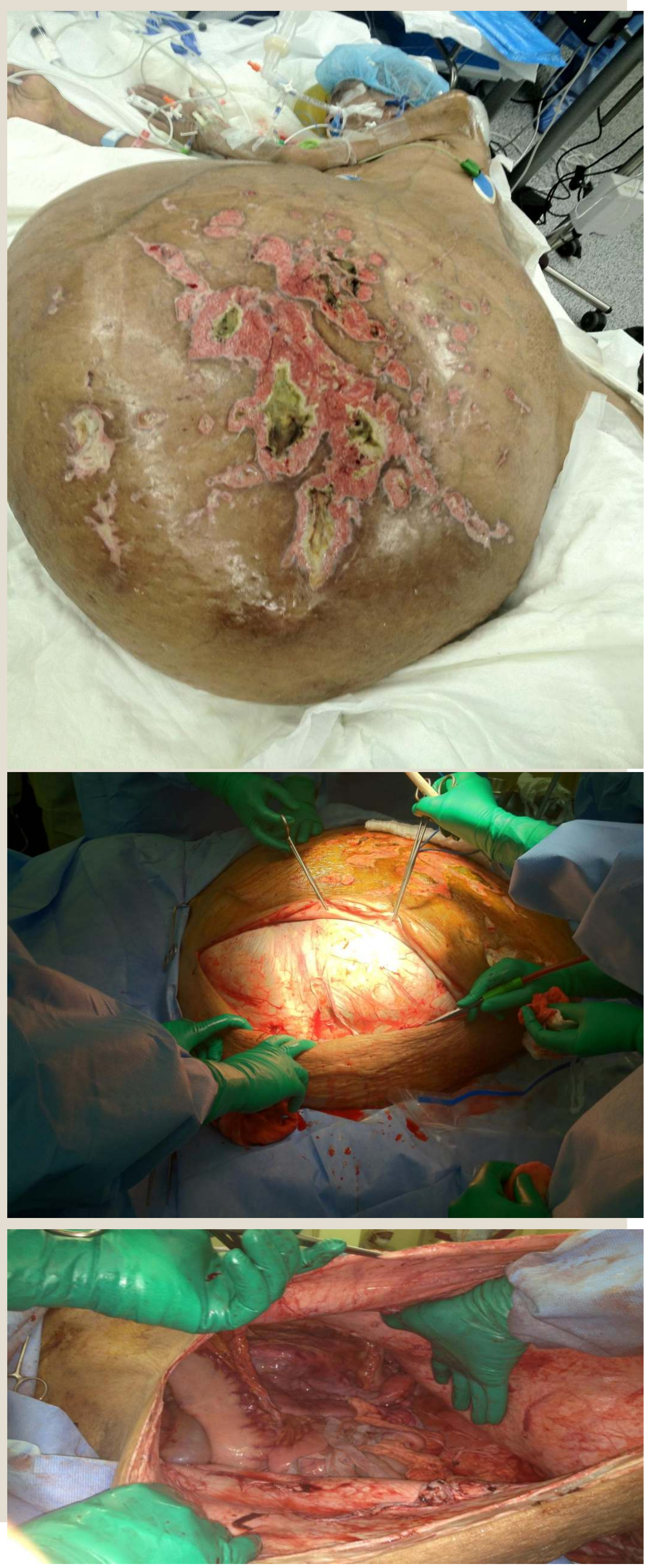